\title{
Tight blood pressure control versus standard blood pressure control on the incidence of myocardial infarction and stroke: A retrospective cohort study
}

\section{Bumsoo Park}

University of Michigan https://orcid.org/0000-0003-4505-2368

Katarzyna Budzynska ( $\sim$ kbudzyn1@hfhs.org)

https://orcid.org/0000-0001-6640-7207

Nada Almasri

Henry Ford Health System

Sumaiya Islam

Henry Ford Health System

\section{Fanar Alyas}

ProMedica Monroe Regional Hospital

Rachel L. Carolan

Henry Ford Health System

Benjamin E. Abraham

Henry Ford Health System

Pamela A. Castro-Camero

Henry Ford Health System

Maria E. Shreve

Henry Ford Health System

Della A. Rees

Henry Ford Health System

Lois Lamerato

Henry Ford Health System

Research article

Keywords: Blood pressure, Myocardial infarction, Stroke

Posted Date: December 4th, 2019

DOI: https://doi.org/10.21203/rs.2.18073/v1 
License: (c) (i) This work is licensed under a Creative Commons Attribution 4.0 International License. Read Full License

Version of Record: A version of this preprint was published at BMC Family Practice on May 16th, 2020. See the published version at https://doi.org/10.1186/s12875-020-01163-4. 


\section{Abstract}

Background The 2017 American College of Cardiology and American Heart Association guideline defined hypertension as blood pressure (BP) $\geq 130 / 80 \mathrm{mmHg}$ compared to the traditional definition of $\geq 140 / 90$ $\mathrm{mmHg}$. This change raised much controversy. We conducted this study to evaluate incidences of myocardial infarction (MI) and stroke comparing tight (TBPC) and standard BP control (SBPC).

Methods The data were collected and analyzed in 2018. We retrospectively identified hypertensive patients for 1 year at our institution who were classified by BP rate across 3 years into 2 groups of TBPC $(<130 \mathrm{mmHg})$ and SBPC $(130-139 \mathrm{mmHg})$. We compared the incidence of new MI and stroke between the 2 groups across a 2-year follow-up. Multivariate analysis was done to identify independent risk factors.

Results Of 5640 study patients, the TBPC group showed significantly less incidence of stroke compared to the SBPC group. No differences were found in MI incidence between the 2 groups. Multivariate analysis showed that increased age independently increased the incidence of both $\mathrm{MI}$ and stroke, and TBPC independently decreased the incidence of stroke but not of MI.

Conclusions Our study showed that TBPC had a significant benefit in less stroke incidence compared to SBPC but did not affect the incidence of MI.

\section{Introduction}

As one of the most common chronic health conditions, hypertension affects more than 1.3 billion adults worldwide [1] and about 75 million adults in the United States [2]. Hypertension can affect major body organs such as heart, brain, kidneys, and eyes and can lead to serious complications such as myocardial infarction (MI), stroke, end-stage renal disease, or visual impairment.

For almost three decades hypertension was defined as systolic blood pressure (SBP) $\geq 140 \mathrm{mmHg}$ or diastolic blood pressure (DBP) $\geq 90 \mathrm{mmHg}$ [3]. Recently, the American College of Cardiology (ACC) and American Heart Association (AHA) published a new guideline defining hypertension as blood pressure (BP) $\geq 130 / 80 \mathrm{mmHg}$, and set the BP treatment goal as $<130 / 80 \mathrm{mmHg}$ [4]. This new guideline was based on the Systolic Blood Pressure Intervention Trial (SPRINT), which in 2015 showed lower rates of fatal and nonfatal major cardiovascular events and all-cause mortality when targeting SBP $<120 \mathrm{mmHg}$ [5].

The new ACC/AHA guideline has raised controversies and the American Academy of Family Physicians recently decided to not endorse it. Because of the controversy over the benefit of tight (TBPC) versus standard BP control (SBPC), we analyzed the incidences of MI and stroke in these 2 patient populations at our large health system.

\section{Methods}




\section{Study Design and Patient Population}

This single-center, retrospective cohort study aimed to assess the occurrence of new $\mathrm{Ml}$ or stroke in patients with TBPC (SBP $<130 \mathrm{mmHg}$ ) versus SBPC (SBP 130-139 mmHg). We addressed only SBP rather than both SBP and DBP based on similar previous studies including the SPRINT trial [5].

The data were collected and analyzed in 2018. We accessed the health system's data stores to identify all outpatient encounters with ICD-10 diagnostic codes for hypertension in 2013 . This resulted in 233,622 encounters in 88,456 patients. From this group we then applied inclusion and exclusion criteria to identify our study patients.

We included patients with SBP $<140 \mathrm{mmHg}$ between ages 40 and 79 years. The age range was based on the recent Centers for Disease Control and Prevention report showing that adults between ages 18 and 44 years mostly had hypertension under control and used antihypertensive medications less often [6]. We excluded patients if the annual SBP measure was absent or if SBP fluctuated between TBPC and SBPC during the 3 years for BP characterization (2013-2015). We excluded patients with diabetes mellitus because the disease is a significant risk factor and a confounder in Ml or stroke outcome. We excluded patients with a history of $\mathrm{MI}$ or stroke before 2013 because these are the highest risk factors for recurrent MI or stroke. We also excluded patients with MI or stroke events between 2013 and 2015. The attrition diagram (Figure 1) summarizes the enrollment process.

\section{Outcome Measures}

The main outcome measures compared between the TBPC and SBPC groups were any new MI or stroke event that occurred within 2 years (2016-2017). Clinical settings of the cardiovascular outcomes included outpatient, emergency department, and inpatient. For ICD-10 coding, we included MI including "late effect" codes, but we did not include angina pectoris. For stroke we counted both ischemic and hemorrhagic strokes and "late effect" codes. We did not include transient ischemic attacks given its potential diagnostic uncertainty. We also excluded traumatic hemorrhages or vascular syndromes (e.g., vertebrobasilar artery syndrome).

\section{Variables}

We obtained baseline demographic data such as age, gender, race, and body mass index (BMI). We noted smoking status, serum low-density lipoprotein (LDL) levels, glomerular filtration rate, aspirin use, antihypertensive use, and statins use. Age was categorized by decade (40-49, 50-59, 60-69, and 70-79 years old). Race/ethnicity was categorized as African American, Asian, Hispanic, white, and other/unknown. For analysis, we further dichotomized race into 2 groups of African American and nonAfrican American based on the recent Centers for Disease Control and Prevention report showing a 
relatively low percentage of hypertension visits among African Americans compared to other races [6]. BMI was categorized as underweight (BMI $<18.5 \mathrm{~kg} / \mathrm{m}^{2}$ ), normal (BMI 18.5-24.9 kg/m²), overweight (BMI $25.0-29.9 \mathrm{~kg} / \mathrm{m}^{2}$ ), obese (BMI $30.0-39.9 \mathrm{~kg} / \mathrm{m}^{2}$ ), and morbidly obese (BMI $\geq 40.0 \mathrm{~kg} / \mathrm{m}^{2}$ ) based on World Health Organization criteria [7]. Smoking status was categorized as "never" or "ever." Serum LDL was categorized as $\geq 190 \mathrm{mg} / \mathrm{dl}$ or $<190 \mathrm{mg} / \mathrm{dl}$; the former is one of the absolute indications of statin use based on 2013 ACC/AHA guidelines [8]. Glomerular filtration rate was categorized as $\geq 30$ $\mathrm{ml} / \mathrm{min} / 1.73 \mathrm{~m}^{2}$ and $<30 \mathrm{ml} / \mathrm{min} / 1.73 \mathrm{~m}^{2}$; the latter is the cutoff value where primary care physicians are recommended to refer patients to nephrology [9]. Use of aspirin, statins, and antihypertensives was determined from medication orders and defined as yes or no.

\section{Adverse Events}

Given the retrospective nature of the study with using a large cohort, we were unable to collect data on the adverse events such as lightheadedness, dizziness, syncope, or falls in the TBPC group. However, we addressed and counted SBP $<90 \mathrm{mmHg}$ in the TBPC group to find out the incidence of significant hypotension.

\section{Statistical Analysis}

Sample characteristics were described using means and standard deviations for continuous variables (SBP) and frequencies (number and percentage) for categorical variables (gender, race, smoking, BMI categories, use of statin, and use of aspirin). To compare the baseline variables between TBPC and SBPC groups, we conducted chi-square tests. For multivariate analysis to determine independent predictors for $\mathrm{MI}$ or stroke incidence, we performed binary logistic regression entering only those variables that showed significant difference between TBPC and SBPC groups with the only exception of the variable 'antihypertensive use versus no use' as this variable was considered to be relevant for the outcomes. All statistical analyses were performed using Epi Info 7 provided by the Centers for Disease Control and Prevention (Atlanta, GA). A P value $<0.05$ was considered statistically significant.

\section{Results}

\section{Baseline Data}

We identified 5640 patients as a final cohort for our study (Figure 1). Significant differences were noted in age, race, BMI, statin use, and the number of antihypertensives between the 2 groups (Table 1). More patients aged 50 to 69 years were in the TBPC group whereas the SBPC group had significantly more patients between 70 and 79 years $(P<0.001)$. African Americans were less likely to have TBPC compared to other races $(P<0.001)$. The TBPC group had more normal weight and overweight patients whereas the SBPC group had significantly more obese and morbidly obese patients $(P<0.001)$. The TBPC group had 
higher statin use than the SBPC group $(P<0.05)$. More patients in the SBPC group used 3 or more antihypertensive medications than those in the TBPC group. No differences were noted in gender, smoking status, serum LDL, glomerular filtration rate, and aspirin use between the 2 groups.

\section{Incidence Outcomes}

The TBPC and SBPC groups showed no difference in incidence of new MI in the 2-year follow-up (Table 2). The SBPC group had a significantly higher incidence of stroke compared to the TBPC group (2.7\% vs. $1.5 \%, P<0.010$ ) (Table 2 ). This gave an absolute risk reduction of $1.2 \%$, and the number needed to treat of approximately 83 .

\section{Multivariate Analysis}

In the logistic regression model, only increased age was an independent predictor of Ml incidence (OR $1.518,95 \% \mathrm{Cl} 1.038-2.219)$ when 'antihypertensive use versus no use' was included as one of the variables (Table 3). However, when 'the number of antihypertensives' was included in the model, increased number of antihypertensives was an independent predictor of MI (OR 2.272, 95\% $\mathrm{Cl} 1.441-$ 3.581) (Table 4). In terms of stroke incidence, both increased age (OR 1.876, 95\% Cl 1.474-2.387) and TBPC (OR 0.583, 95\% Cl 0.374-0.910) were significantly associated with stroke incidence when 'antihypertensive use versus no use' was included as one of the variables (Table 3). When 'the number of antihypertensives' was included in the model, increased age (OR 1.792, 95\% Cl 1.406-2.284), increased number of antihypertensives (OR 1.282, 95\% Cl 1.011-1.627), and TBPC (OR 0.589, 95\% CI 0.377-0.919) were independent predictors of stroke (Table 4).

\section{Adverse Events}

Among the 4530 patients in the TBPC group, only 5 patients $(0.001 \%)$ had SBP $<90 \mathrm{mmHg}$.

\section{Discussion}

This single-center cohort study of more than 5000 hypertensive patients evaluated the impact of TBPC $(\mathrm{SBP}<130 \mathrm{mmHg})$ on the incidence of new MI and stroke. We found that TBPC independently decreased the incidence of stroke compared to SBPC, but did not have an impact on MI incidence.

A retrospective study using the ACTION trial database found the greatest difference between SBP $<130$ $\mathrm{mmHg}$ and SBP $<140 \mathrm{mmHg}$ groups in the risk of stroke [10]. The cohort in the ACTION trial was different from ours in that their patients had stable coronary heart disease [11]. A meta-analysis performed by Lee et al demonstrated that SBP < $130 \mathrm{mmHg}$ compared to SBP 130-139 mmHg showed additional protective effect on stroke in patients with cardiovascular risk factors but no previously 
established cardiovascular disease [12]. In another meta-analysis, Reboldi et al found that more-tight SBP control (<120 mmHg), compared to less-tight SBP control ( $<140 \mathrm{mmHg})$, significantly reduced stroke risk by $31 \%$, but no significant risk reduction was noted in MI [13]. While these results are similar to ours, Reboldi et al's analysis included diabetic patients and a different BP cutoff for the 2 groups. The ONTARGET trial provided further evidence on the significant impact of BP control on stroke but not on MI [14]. The trial found that more frequent achievement of target BPs $(<130 / 90 \mathrm{mmHg}$ or $<140 / 90 \mathrm{mmHg})$ resulted in significant cerebrovascular protection but not cardiac protection [14].

Why TBPC decreases the risk of stroke but not MI remains unclear. Several long-term prospective cohort studies have shown that hypertension increased the relative risk of stroke to a greater degree than $\mathrm{MI}$ [15-17]. One possible hypothesis is different blood flow physiology between the brain and heart. The brain receives a larger fraction of cardiac output by nearly 3-fold than the heart [18]. While most of the coronary blood flow occurs during diastole, the cerebral blood flow occurs during systole [19]. Therefore, cerebral blood flow might be more sensitive to a change in SBP than coronary blood flow, and coronary blood flow might be more sensitive to a change in DBP than SBP. A recent multinational study supports this hypothesis in that results showed increased risk of MI with increased DBP whereas increased SBP did not increase the risk of MI [20].

Other studies have reported different results. Verdecchia et al performed an open-label randomized trial and found no difference in the incidence of Ml or stroke between TBPC $(<130 \mathrm{mmHg})$ and SBPC $(<140$ $\mathrm{mmHg}$ ) [21]. Their population was similar to ours in the exclusion of diabetic patients. Another large randomized study using the database of the Hypertension Optimal Treatment trial showed that TBPC (< $130 \mathrm{mmHg}$ ) in patients with diabetes and coronary artery disease did not improve cardiovascular outcomes compared to SBPC ( $<140 \mathrm{mmHg}$ ) [22]. The ACCORD study conducted in 2010 also showed no difference in fatal and nonfatal cardiovascular events between TBPC $(<120 \mathrm{mmHg})$ and SBPC $(<140$ $\mathrm{mmHg}$ ) groups in diabetic patients. More importantly, the TBPC group demonstrated significantly higher rates of serious adverse events attributed to antihypertensive therapy [23]. Furthermore, a post hoc analysis using the Irbesartan Diabetic Nephropathy Trial database concluded that BP $\leq 120 / 85 \mathrm{mmHg}$ may be associated with increased cardiovascular events [24].

Our study and the majority of cohort and prospective studies have shown that TBPC is associated with decreased risk of stroke. Nevertheless, the issue of TBPC must be addressed carefully in the clinical setting. Even though cerebral blood flow has an autoregulation mechanism, the mechanism can be lost if the mean arterial BP drops below $60 \mathrm{mmHg}$ [25]. A study that targeted $\mathrm{BP}<130 / 80 \mathrm{mmHg}$ for diabetic patients found intensive BP control caused progressive reduction of cerebral blood flow velocity [26]. Ferreira et al recently demonstrated significantly increased rates of cardiovascular death, heart failure hospitalization, MI, and stroke when SBP decreased below $125 \mathrm{mmHg}$ [20]. In our study, only $0.001 \%$ $(5 / 4530)$ in the TBPC group showed SBP $<90 \mathrm{mmHg}$.

An interesting finding in our study is that statin use did not independently affect $\mathrm{Ml}$ or stroke incidence. Based on the 2013 ACC/AHA guidelines, absolute indications for statin use include clinical 
atherosclerotic cardiovascular disease, type 2 diabetes mellitus, and serum LDL $\geq 190 \mathrm{mg} / \mathrm{dl}$ [8]. Perhaps statin use did not affect the Ml or stroke incidence in our study because we excluded patients with diabetes and those with a history of Ml or stroke. Very few patients in our study had a serum LDL $\geq 190$ $\mathrm{mg} / \mathrm{dl}$ (Table 1). Another issue is we only counted the number of medication orders rather than addressing the actual number of medications taken. So it is unclear whether the medications were actually dispensed and taken. Another interesting finding is that increased number of antihypertensive medications significantly predicted both $\mathrm{MI}$ and stroke incidence while the usage of antihypertensive medication itself did not predict either MI or stroke incidence in the multivariate model. This can suggest that requiring more antihypertensive medications rather than taking the medications itself could predict higher cardiovascular risk.

\section{Limitations}

Our study has several limitations. Because of the retrospective design, we were not able to completely address and remove all potential confounders. There were significant differences in age, race, $\mathrm{BMI}$, statin use, and the number of antihypertensives between TBPC and SBPC groups. The TBPC group was younger and had statins prescribed more often than the SBPC group. Although these variables were adjusted at multivariate analysis, these differences might still have affected our findings. As we excluded other significant risk factors of $\mathrm{MI}$ and stroke, such as diabetes and history of Ml or stroke, our study population was different from actual patients commonly seen in clinical practice, which might have resulted in the relatively rare outcome events in our study. Also, we were not able to collect information on the adverse events of TBPC.

\section{Conclusion}

Our retrospective cohort study showed that TBPC had a significant benefit in less stroke incidence compared to SBPC, although it did not affect the incidence of MI. Our study could help aid in the discussion regarding the association of BP control and stroke in actual clinical practice. Even though the American Academy of Family Physicians decided to not endorse the ACC/AHA guideline, our study shows more favorable result towards the SPRINT trial in terms of less stroke incidence.

\section{Abbreviations}

ACC: American College of Cardiology; AHA: American Heart Association; BMI: Body mass index; BP: Blood pressure; DBP: Diastolic blood pressure; LDL: Low-density lipoprotein; MI: Myocardial infarction; SBP: Systolic blood pressure; SBPC: Standard blood pressure control; SPRINT: Systolic Blood Pressure Intervention Trial; TBPC: Tight blood pressure control

\section{Declarations}

\section{Ethics approval and consent to participate}


The study protocol was approved by the institutional review Board of Henry Ford Health System (IRB No. 11950) under expedited review with waiver of informed consent.

\section{Consent for publication}

Not applicable.

\section{Availability of data and materials}

The datasets used and/or analyzed during the current study are available from the corresponding author on reasonable request.

\section{Competing interests}

All authors declare no competing interests for this study.

\section{Funding}

There was no funding support applicable to the study.

\section{Authors' contributions}

All authors designed and conceived of the study as a group project. BP drafted the manuscript. BP, NA, SI, FA, RC, BEA, PACC, and LL collected and analyzed the data. LL performed a statistical analysis. KB, MES, DAR, and LL supervised overall study process, and revised the manuscript. All authors read and approved the final manuscript.

\section{Acknowledgements}

This study was conducted as an annual scholarly project of the family medicine residency program at Henry Ford Health System in 2018. This study was presented at Family Medicine Experience (FMX) 2018 in New Orleans, Louisiana.

\section{References}

1. Bloch MJ. Worldwide prevalence of hypertension exceeds 1.3 billion. J Am Soc Hypertens. 2016;10(10):753-4. https://doi.org/10.1016/j.jash.2016.08.006. 
2. Merai R, Siegel C, Rakotz M, et al. CDC Grand Rounds: a public health approach to detect and control hypertension. MMWR Morb Mortal Wkly Rep. 2016;65(45):1261-4. https://doi.org/10.15585/mmwr.mm6545a3.

3. Chobanian AV, Alderman MH, DeQuattro V, et al. The 1988 Report of the Joint National Committee on Detection, Evaluation, and Treatment of High Blood Pressure. Arch Intern Med. 1988;148(5):102338.

4. Whelton PK, Carey RM, Aronow WS, et al. 2017 ACC/AHA/AAPA/ABC/ACPM/AGS/APhA/ASH/ASPC/NMA/PCNA guideline for the prevention, detection, evaluation, and management of high blood pressure in adults: a report of the American College of Cardiology/American Heart Association Task Force on Clinical Practice Guidelines. Hypertension. 2018;71(6):1269-324. https://doi.org/10.1161/HYP.0000000000000065.

5. SPRINT Research Group, Wright JT Jr, Williamson JD, et al. A randomized trial of intensive versus standard blood-pressure control. N Engl J Med. 2015;373(22):2103-16. https://doi.org/10.1056/NEJMoa1511939.

6. Ashman JJ, Rui P, Schappert SM, Strashny A. Characteristics of visits to primary care physicians by adults diagnosed with hypertension. Natl Health Stat Report. 2017;(106):1-14.

7. National Heart Lung and Blood Institute Obesity Education Initiative Expert Panel on the Identification, Evaluation, and Treatment of Obesity in Adults. Clinical Guidelines on the Identification, Evaluation, and Treatment of Overweight and Obesity in Adults: The Evidence Report. Obes Res. 1998;6(Suppl 2):51S-209S.

8. Stone NJ, Robinson JG, Lichtenstein AH, et al. 2013 ACC/AHA guideline on the treatment of blood cholesterol to reduce atherosclerotic cardiovascular risk in adults: a report of the American College of Cardiology/American Heart Association Task Force on Practice Guidelines. Circulation. 2014;129(25 Suppl 2):S1-S45. https://doi.org/10.1161/01.cir.0000437738.63853.7a.

9. Inker LA, Astor BC, Fox CH, et al. KDOQI US commentary on the $2012 \mathrm{KDIGO}$ clinical practice guideline for the evaluation and management of CKD. Am J Kidney Dis. 2014;63(5):713-35. https://doi.org/10.1053/j.ajkd.2014.01.416.

10. Meredith PA, Lloyd SM, Ford I, Elliott HL. Importance of sustained and "tight" blood pressure control in patients with high cardiovascular risk. Blood Press. 2016;25(2):74-82. https://doi.org/10.3109/08037051.2015.1127528.

11. Poole-Wilson PA, Lubsen J, Kirwan BA, et al. Effect of long-acting nifedipine on mortality and cardiovascular morbidity in patients with stable angina requiring treatment (ACTION trial): randomised controlled trial. Lancet. 2004;364(9437):849-57.

12. Lee M, Saver JL, Hong KS, Hao Q, Ovbiagele B. Does achieving an intensive versus usual blood pressure level prevent stroke? Ann Neurol. 2012;71(1):133-40. https://doi.org/10.1002/ana.22496.

13. Reboldi G, Gentile G, Angeli F, Ambrosio G, Mancia G, Verdecchia P. Effects of intensive blood pressure reduction on myocardial infarction and stroke in diabetes: a meta-analysis in 73,913 patients. J Hypertens. 2011;29(7):1253-69. https://doi.org/10.1097/HJH.0b013e3283469976. 
14. Mancia G, Schumacher H, Redon J, et al. Blood pressure targets recommended by guidelines and incidence of cardiovascular and renal events in the Ongoing Telmisartan Alone in Combination with Ramipril Global Endpoint Trial (ONTARGET). Circulation. 2011;124(16):1727-36. https://doi.org/10.1161/CIRCULATIONAHA.110.008870.

15. Nielsen WB, Vestbo J, Jensen GB. Isolated systolic hypertension as a major risk factor for stroke and myocardial infarction and an unexploited source of cardiovascular prevention: a prospective population-based study. J Hum Hypertens. 1995;9(3):175-80.

16. Kannel WB. Blood pressure as a cardiovascular risk factor: prevention and treatment. JAMA. 1996;275(20):1571-6.

17. Antikainen R, Jousilahti P, Tuomilehto J. Systolic blood pressure, isolated systolic hypertension and risk of coronary heart disease, strokes, cardiovascular disease and all-cause mortality in the middleaged population. J Hypertens. 1998;16(5):577-83.

18. Sidhu P, Peng HT, Cheung B, Edginton A. Simulation of differential drug pharmacokinetics under heat and exercise stress using a physiologically based pharmacokinetic modeling approach. Can $\mathrm{J}$ Physiol Pharmacol. 2011;89(5):365-82. https://doi.org/10.1139/y11-030.

19. Duncker DJ, Merkus D. Regulation of coronary blood flow. Effect of coronary artery stenosis. Arch Mal Coeur Vaiss. 2004;97(12):1244-50.

20. Ferreira JP, Duarte K, Pfeffer MA, et al. Association between mean systolic and diastolic blood pressure throughout the follow-up and cardiovascular events in acute myocardial infarction patients with systolic dysfunction and/or heart failure: an analysis from the High-Risk Myocardial Infarction Database Initiative. Eur J Heart Fail. 2018;20(2):323-31. https://doi.org/10.1002/ejhf.1131.

21. Verdecchia P, Staessen JA, Angeli F, et al. Usual versus tight control of systolic blood pressure in nondiabetic patients with hypertension (Cardio-Sis): an open-label randomised trial. Lancet. 2009;374(9689):525-33. https://doi.org/10.1016/S0140-6736(09)61340-4.

22. Cooper-DeHoff RM, Gong Y, Handberg EM, et al. Tight blood pressure control and cardiovascular outcomes among hypertensive patients with diabetes and coronary artery disease. JAMA. 2010;304(1):61-8. https://doi.org/10.1001/jama.2010.884.

23. ACCORD Study Group, Cushman WC, Evans GW, et al. Effects of intensive blood-pressure control in type 2 diabetes mellitus. N Engl J Med. 2010;362(17):1575-85. https://doi.org/10.1056/NEJMoa1001286.

24. Berl T, Hunsicker LG, Lewis JB, et al. Impact of achieved blood pressure on cardiovascular outcomes in the Irbesartan Diabetic Nephropathy Trial. J Am Soc Nephrol. 2005;16(7):2170-9. https://doi.org/10.1681/ASN.2004090763.

25. Paulson OB, Strandgaard S, Edvinsson L. Cerebral autoregulation. Cerebrovasc Brain Metab Rev. 1990;2(2):161-92.

26. Kim YS, Davis SC, Truijen J, et al. Intensive blood pressure control affects cerebral blood flow in type 2 diabetes mellitus patients. Hypertension. 2011;57(4):738-45. https://doi.org/10.1161/HYPERTENSIONAHA.110.160523. 


\section{Tables}

Table 1. Baseline data for patients with tight versus standard blood pressure control 


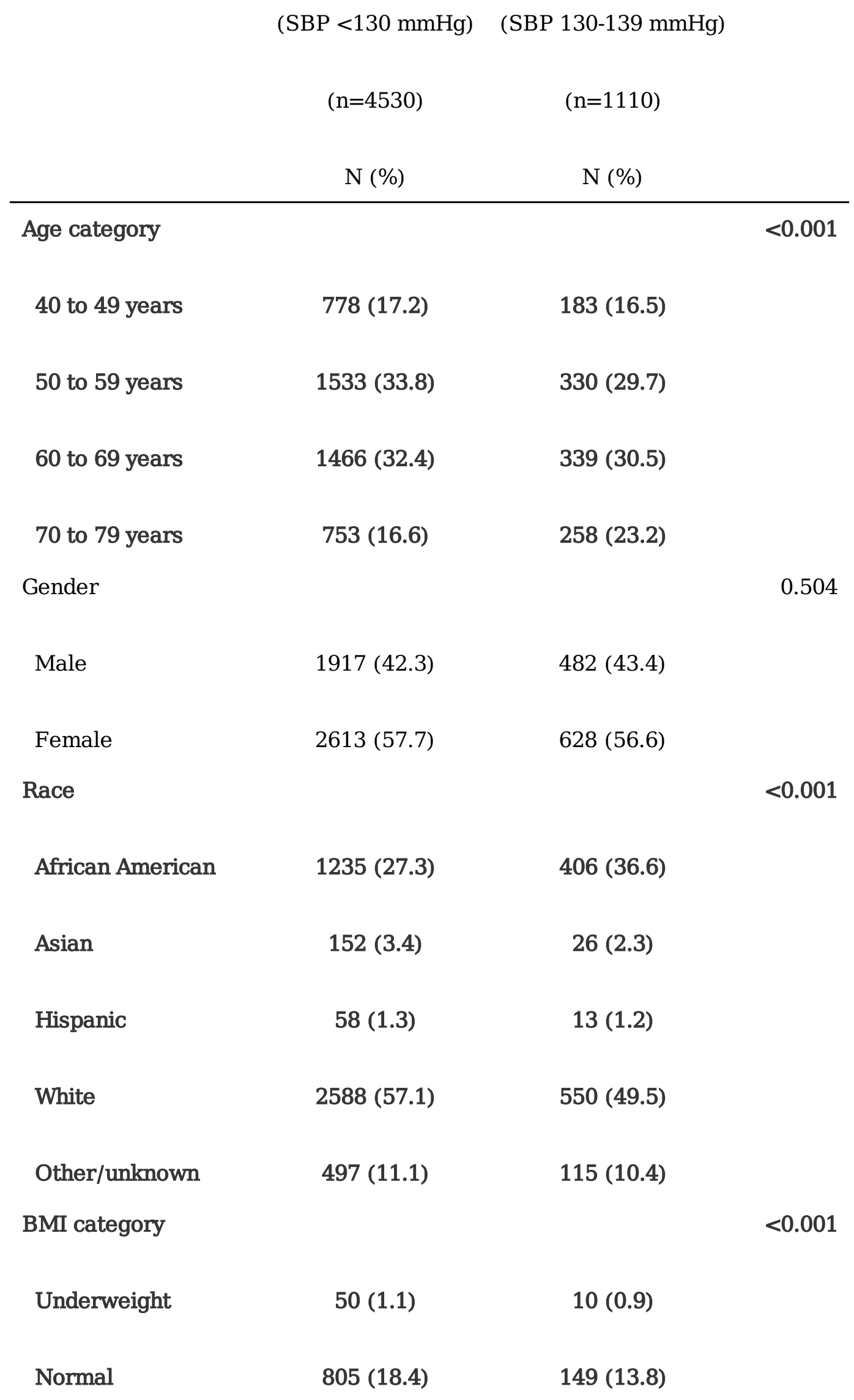




\section{Overweight}

Obese

Morbidly obese

Smoking

Ever

Never

Serum LDL level

$\geq 190 \mathrm{mg} / \mathrm{dl}$

$<190 \mathrm{mg} / \mathrm{dl}$

EGFR (race-adjusted)

$\geq 30 \mathrm{ml} / \mathrm{min} / 1.73 \mathrm{~m}^{2}$

$<30 \mathrm{ml} / \mathrm{min} / 1.73 \mathrm{~m}^{2}$

Aspirin use

Yes

No

Statin use

Yes

No

Antihypertensive use

Yes

No

No. Antihypertensives

0
1602 (36.6)

1624 (37.1)

293 (6.7)

109 (10.1)

467 (43.4)

0.399

2195 (48.5)

2333 (51.5)

586 (52.9)

$521(47.1)$

0.182

$22(0.6)$

3652 (99.4)

894 (99.0)

9 (1.0)

905 (99.6)

$4(0.4)$

0.333

3653 (99.3)

$27(0.7)$

0.529

1073 (23.7)

253 (22.8)

3457 (76.3)

857 (77.2)

$<0.05$

2008 (44.3)

450 (40.5)

2522 (55.7)

660 (59.5)

0.646

4328 (95.5)

1064 (95.9)

202 (4.5)

46 (4.1)

$<0.001$

202 (4.5)

46 (4.1)

347 (31.3) 
${ }^{\mathrm{a} C h i-s q u a r e ~ t e s t . ~}$

Boldface indicates statistical significance $(\mathrm{p}<0.05)$.

Table 2. Two-year cardiovascular incidence in tight versus standard blood pressure control

\begin{tabular}{lccc}
\hline Variables & TBPC & SBPC & $P$ value $^{\mathrm{a}}$ \\
& $(\mathrm{SBP}<130 \mathrm{mmHg})$ & $(\mathrm{SBP} 130-139 \mathrm{mmHG})$ & \\
& $\mathrm{N}(\%)$ & $(\mathrm{n}=1110)$ & \\
& & $\mathrm{N}(\%)$ & 0.476 \\
Myocardial infarction & & & \\
Yes & $28(0.6)$ & $9(0.8)$ & \\
No & $4502(99.4)$ & $1101(99.2)$ & \\
Stroke & & $30(2.7)$ & \\
Yes & $68(1.5)$ & $1080(97.3)$ & \\
No & $4462(98.5)$ & & \\
\hline
\end{tabular}

${ }^{\mathrm{a} C h i-s q u a r e}$ test.

Boldface indicates statistical significance $(\mathrm{p}<0.05)$.

Table 3. Multivariate analysis to predict myocardial infarction and stroke 


\begin{tabular}{llc}
\hline Variables & OR (95\% CI) & $P$ value $^{\mathrm{a}}$ \\
\hline Myocardial infarction & & \\
\hline Age & $1.518(1.038-2.219)$ & 0.032 \\
African American (vs. other races) & $1.010(0.464-2.199)$ & 0.981 \\
Body mass index & $0.828(0.562-1.219)$ & 0.338 \\
Statin use (vs. no use) & $1.408(0.701-2.829)$ & 0.314 \\
Antihypertensive use (vs. no use) & $140232.7426(0.000->1.0 \mathrm{E} 12)$ & 0.969 \\
Tight (vs. standard) BP control & $0.734(0.339-1.588)$ & 0.432 \\
\hline Stroke & $1.876(1.474-2.387)$ & $<0.001$ \\
\hline Age & $1.080(0.677-1.722)$ & 0.748 \\
African American (vs. other races) & $0.940(0.742-1.192)$ & 0.610 \\
Body mass index & $1.338(0.876-2.044)$ & 0.178 \\
Statin use (vs. no use) & $1.911(0.466-7.838)$ & 0.369 \\
Antihypertensive use (vs. no use) & $0.583(0.374-0.910)$ & 0.018 \\
\hline Tight (vs. standard) BP control & & \\
\hline
\end{tabular}

ainary logistic regression test.

Boldface indicates statistical significance $(\mathrm{p}<0.05)$.

Table 4. Multivariate analysis to predict myocardial infarction and stroke (Number of antihypertensives as one of the variables) 


\begin{tabular}{lcc}
\hline Variables & OR (95\% CI) & $P$ value $^{\mathrm{a}}$ \\
\hline Myocardial infarction & $1.358(0.929-1.986)$ & 0.115 \\
\hline Age & $0.910(0.416-1.991)$ & 0.814 \\
African American (vs. other races) & $0.960(0.904-1.019)$ & 0.176 \\
Body mass index & $1.269(0.630-2.557)$ & 0.504 \\
Statin use (vs. no use) & $2.272(1.441-3.581)$ & $<0.001$ \\
Number of antihypertensives & $0.788(0.363-1.707)$ & 0.545 \\
Tight (vs. standard) BP control & $1.792(1.406-2.284)$ & $<0.001$ \\
\hline Stroke & $1.059(0.663-1.690)$ & 0.812 \\
\hline Age & $0.979(0.944-1.015)$ & 0.243 \\
African American (vs. other races) & $1.300(0.849-1.990)$ & 0.227 \\
Body mass index & $1.282(1.011-1.627)$ & 0.040 \\
Statin use (vs. no use) & $0.589(0.377-0.919)$ & 0.020 \\
Number of antihypertensives & & \\
Tight (vs. standard) BP control & & \\
\hline
\end{tabular}

${ }^{a}$ Binary logistic regression test.

Boldface indicates statistical significance $(\mathrm{p}<0.05)$.

\section{Figures}




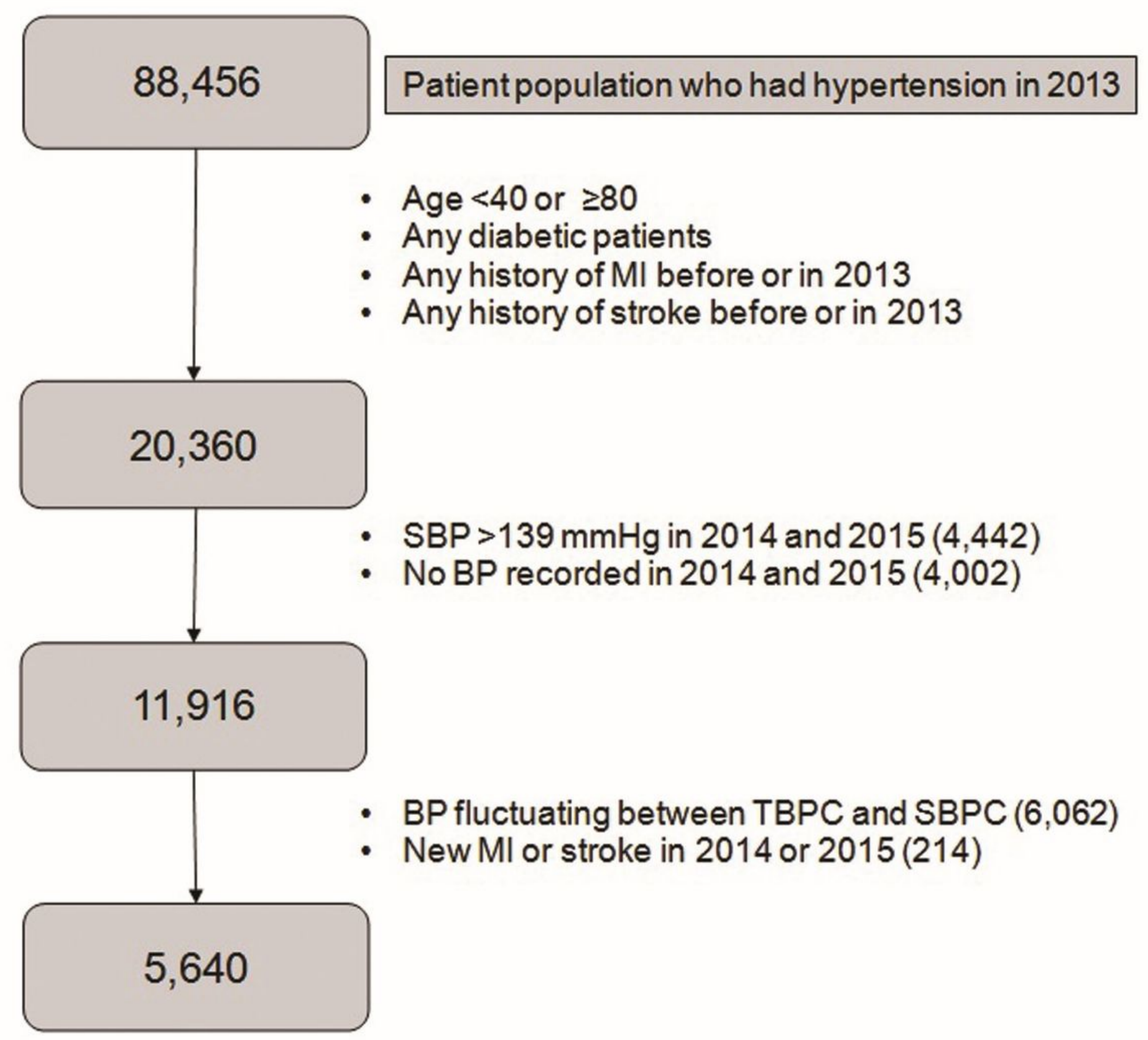

Figure 1

Attrition diagram of the study population. 